\title{
Research Article \\ On a Suzuki Type General Fixed Point Theorem with Applications
}

\author{
S. L. Singh, ${ }^{1,2}$ H. K. Pathak, ${ }^{1,3}$ and S. N. Mishra ${ }^{1}$ \\ ${ }^{1}$ Department of Mathematics, Walter Sisulu University, Mthatha 5117, South Africa \\ 221 Govind Nagar, Rishikesh 249201, India \\ ${ }^{3}$ School of Studies in Mathematics, Pt. Ravishankar Shukla University, Raipur 492010, India \\ Correspondence should be addressed to S. N. Mishra, smishra@wsu.ac.za
}

Received 29 October 2010; Accepted 2 December 2010

Academic Editor: A. T. M. Lau

Copyright $@ 2010$ S. L. Singh et al. This is an open access article distributed under the Creative Commons Attribution License, which permits unrestricted use, distribution, and reproduction in any medium, provided the original work is properly cited.

The main result of this paper is a fixed-point theorem which extends numerous fixed point theorems for contractions on metric spaces and recently developed Suzuki type contractions. Applications to certain functional equations and variational inequalities are also discussed.

\section{Introduction}

The classical Banach contraction theorem has numerous generalizations, extensions, and applications. In a comprehensive comparison of contractive conditions, Rhoades [1] recognized that Ćirićs quasicontraction [2] (see condition (C) below) is the most general condition for a self-map $T$ of a metric space which ensures the existence of a unique fixed point. Pal and Maiti [3] proposed a set of conditions (see (PM.1)-(PM.4) below) as an extension of the principle of quasicontraction (C), under which $T$ may have more than one fixed point (see Example 2.7 below). Thus the condition (C) is independent of the conditions (PM.1)-(PM.4) (see also Rhoades [4, page 42]).

On the other hand, Suzuki [5] recently obtained a remarkable generalization of the Banach contraction theorem which itself has been extended and generalized on various settings (see, e.g, [6-15]). With a view of extending Suzuki's contraction theorem [5] and its several generalizations, we combine the ideas of Pal and Maiti [3], Suzuki [5], and Popescu [10] to obtain a very general fixed-point theorem. Subsequently, we use our results to solve certain functional equations and variational inequalities under different conditions than those considered in Bhakta and Mitra [16], Baskaran and Subrahmanyam [17], Pathak et al. [18, 19], Singh and Mishra [11, 12], and Pathak et al. [20, and references thereof]. 

$x, y \in X:$

Consider the following conditions for a map $T$ from a metric space $(X, d)$ to itself for

(C) $d(T x, T y) \leq k \max \{d(x, y), d(x, T x), d(y, T y), d(x, T y), d(y, T x)\}, 0<k<1$,

(PM.1) $d(x, T x)+d(y, T y) \leq a d(x, y), 1<a<2$,

(PM.2) $d(x, T x)+d(y, T y) \leq b[d(x, T y)+d(y, T x)+d(x, y)], 1 / 2<b<2 / 3$,

(PM.3) $d(x, T x)+d(y, T y)+d(T x, T y) \leq c[d(x, T y)+d(y, T x)], 1<c<3 / 2$,

(PM.4) $d(T x, T y) \leq k \max \{d(x, y), d(x, T x), d(y, T y),(1 / 2)[d(x, T y), d(y, T x)]\}, 0<k<1$.

\section{Main Results}

Throughout this paper, we denote by $\mathbb{N}$ the set of natural numbers. We suppose that

$$
\eta=\min \left\{\frac{1}{a}, \frac{1-b}{3 b}, \frac{2-c}{2 c-1}, \frac{1}{1+k}\right\}
$$

where $a, b, c$, and $k$ are as in conditions (PM.1)-(PM.4).

Notice that

$$
\begin{gathered}
\frac{1}{2}<\frac{1}{a}<1, \quad \frac{1}{6}<\frac{1-b}{3 b}<\frac{1}{3} \\
\frac{1}{4}<\frac{2-c}{2 c-1}<1, \quad \frac{1}{2}<\frac{1}{1+k}<1 .
\end{gathered}
$$

Evidently, $\eta(1+k) \leq 1$.

An orbit $O\left(T, x_{0}\right)$ of $T: X \rightarrow X$ at $x_{0} \in X$ is a sequence $\left\{x_{n}: x_{n}=T^{n} x_{0}, n=1,2, \ldots\right\}$. A space $X$ is $T$-orbitally complete if and only if every Cauchy sequence contained in the orbit $O\left(T, x_{0}\right)$ converges in $X$, for all $x_{0} \in X$.

An orbit of a multivalued map $P: X \rightarrow 2^{X}$, the collection of nonempty subsets of $X$, at $x_{0} \in X$ is a sequence $\left\{x_{n}: x_{n} \in P x_{n-1}, n=1,2, \ldots\right\}$. X is called $P$-orbitally complete if every Cauchy sequence of the form $\left\{x_{n_{i}}: x_{n_{i}} \in P x_{n_{i}-1}, i=1,2, \ldots\right\}$ converges in $X$, for all $x_{0} \in X$. For details, refer to Ćirić $[2,21]$.

The following theorem is our main result.

Theorem 2.1. Let $T$ be a self-map of a metric space $X$ and $X$ be $T$-orbitally complete. Assume that there exists an $x_{0} \in X$ such that for any two elements $x, y \in \overline{O\left(T, x_{0}\right)}$,

$$
\eta d(x, T x) \leq d(x, y)
$$

implies that at least one of the conditions (PM.1), (PM.2), (PM.3), and (PM.4) is true. Then, the sequence $\left\{T^{n} x_{0}\right\}$ converges in $X$ and $z=\lim _{n \rightarrow \infty} T^{n} x_{0}$ is a fixed point of $T$. 
Proof. Define a sequence $\left\{d_{n}\right\}$ such that $d_{n}=d\left(x_{n}, x_{n+1}\right)$, where $x_{n}=T^{n} x_{0}, n \in \mathbb{N}$. Since $\eta d\left(x_{n}, T x_{n}\right) \leq d\left(x_{n}, T x_{n}\right)$ for any $n \in \mathbb{N}$, one of the conditions (PM.1)-(PM.4) is true for the pair $x_{n}, x_{n+1}$. If (PM.1) is true, then

$$
d\left(x_{n}, x_{n+1}\right)+d\left(x_{n+1}, x_{n+2}\right) \leq a d\left(x_{n}, x_{n+1}\right) .
$$

This yields

$$
d_{n+1} \leq(a-1) d_{n}
$$

Similarly, if (PM.2), (PM.3), and (PM.4) are true, then correspondingly we obtain

$$
\begin{gathered}
d_{n+1} \leq \frac{2 b-1}{1-b} d_{n} \\
d_{n+1} \leq \frac{c-1}{2-c} d_{n} \\
d_{n+1} \leq k d_{n} .
\end{gathered}
$$

Hence, from (2.5)-(2.6),

$$
d_{n+1} \leq \lambda d_{n}
$$

where

$$
\lambda=\max \left\{a-1, \frac{2 b-1}{1-b}, \frac{c-1}{2-c}, k\right\} .
$$

Since $0<\lambda<1$, the sequence $\left\{x_{n}\right\}$ is Cauchy. By the $T$-orbital completeness of $X$, the limit $z$ of the sequence $\left\{x_{n}\right\}$ is in $X$. Moreover, there exists $n_{0} \in \mathbb{N}$ such that

$$
\eta d\left(x_{n}, T x_{n}\right) \leq d\left(x_{n}, x\right)
$$

for $n \geq n_{0}$, where $x \neq z$. Therefore, by conditions (PM.1)-(PM.4), we have one of the following for $x \neq z$ :

$$
d\left(x_{n}, T x_{n}\right)+d(x, T x) \leq a d\left(x_{n}, x\right),
$$

which yields on making $n \rightarrow \infty$,

$$
d(x, T x) \leq \operatorname{ad}(x, z)
$$


and similarly

$$
\begin{gathered}
d(x, T x) \leq \frac{3 b}{1-b} d(x, z), \\
d(x, T x) \leq \frac{2 c-1}{2-c} d(x, z), \\
d(z, T x) \leq k \max \{d(x, z), d(x, T x)\},
\end{gathered}
$$

that is,

$$
d(z, T x) \leq k d(x, T x)
$$

or

$$
d(z, T x) \leq k d(x, z)
$$

and in this case

$$
d(x, T x) \leq d(x, z)+d(z, T x) \leq d(x, z)+k d(x, z),
$$

that is,

$$
\frac{1}{1+k} d(x, T x) \leq d(x, z) .
$$

Thus, in view of (2.11), (2.12), (2.13), (2.18), and (2.15), one of the following is true for $x \neq z$ :

$$
\begin{gathered}
\eta d(x, T x) \leq d(x, z), \\
d(z, T x) \leq k d(x, T x) .
\end{gathered}
$$

Case 1. Suppose that (2.19) is true. Then, by the assumption, one of (PM.1)-(PM.4) is true, that is,

$$
\begin{gathered}
d(x, T x)+d(z, T z) \leq a d(x, z), \\
d(x, T x)+d(z, T z) \leq b[d(x, T z)+d(z, T x)+d(x, z)], \\
d(x, T x)+d(z, T z)+d(T x, T z) \leq c[d(x, T z)+d(z, T x)], \\
d(T x, T z) \leq k \max \left\{d(x, z), d(x, T x), d(z, T z), \frac{1}{2}[d(x, T z)+d(z, T x)]\right\} .
\end{gathered}
$$


Taking $x=x_{n}$ in these inequaliteis and making $n \rightarrow \infty$, we see that one of the following is true:

$$
d(z, T z) \leq 0, \quad(1-b) d(z, T z) \leq 0, \quad(2-c) d(z, T z) \leq 0, \quad(1-k) d(z, T z) \leq 0 .
$$

All these possibilities lead to the fact that $T z=z$.

Case 2. Suppose that (2.20) is true. We show that there exists a subsequence $\left\{n_{j}\right\}$ of $\{n\}$ such that

$$
\eta d\left(x_{n_{j}}, x_{n_{j}+1}\right) \leq d\left(x_{n_{j}}, z\right), \quad j \in \mathbb{N} .
$$

Recall that by (2.7),

$$
d\left(x_{n}, x_{n+1}\right) \leq \lambda d\left(x_{n-1}, x_{n}\right)
$$

Suppose that

$$
\eta d\left(x_{n-1}, x_{n}\right)>d\left(x_{n-1}, z\right), \quad \eta d\left(x_{n}, x_{n+1}\right)>d\left(x_{n}, z\right) .
$$

Then

$$
\begin{aligned}
d\left(x_{n-1}, x_{n}\right) & \leq d\left(x_{n-1}, z\right)+d\left(x_{n}, z\right) \\
& <\eta d\left(x_{n-1}, x_{n}\right)+\eta d\left(x_{n}, x_{n+1}\right) \\
& \leq \eta d\left(x_{n-1}, x_{n}\right)+\eta \lambda d\left(x_{n-1}, x_{n}\right) \\
& =\eta(1+\lambda) d\left(x_{n-1}, x_{n}\right) .
\end{aligned}
$$

Since without loss of generality, we may take $\lambda=k$, we have

$$
\begin{aligned}
d\left(x_{n-1}, x_{n}\right) & <\eta(1+k) d\left(x_{n-1}, x_{n}\right) \\
& \leq d\left(x_{n-1}, x_{n}\right) .
\end{aligned}
$$

This is a contradiction. Therefore, either

$$
\eta d\left(x_{n-1}, x_{n}\right) \leq d\left(x_{n-1}, z\right), \quad \text { or } \quad \eta d\left(x_{n}, x_{n+1}\right) \leq d\left(x_{n}, z\right) .
$$

This implies that either

$$
\eta d\left(x_{2 n-1}, x_{2 n}\right) \leq d\left(x_{2 n-1}, z\right), \quad \text { or } \quad \eta d\left(x_{2 n}, x_{2 n+1}\right) \leq d\left(x_{2 n}, z\right)
$$


holds for $n \in \mathbb{N}$. Thus, there exists a subsequence $\left\{n_{j}\right\}$ of $\{n\}$ such that

$$
\eta d\left(x_{n_{j}}, x_{n_{j}+1}\right) \leq d\left(x_{n_{j}}, z\right)
$$

that is,

$$
\eta d\left(x_{n_{j}}, T x_{n_{j}}\right) \leq d\left(x_{n_{j}}, z\right) \text { for } j \in \mathbb{N}
$$

Hence, by the assumption, one of the conditions (PM.1)-(PM.4) is satisfied for $x=x_{n_{j}}$ and $y=z$, and making $j \rightarrow \infty$, we obtain $z=T z$.

Remark 2.2. If only the condition (PM.4) is satisfied in Theorem 2.1, then the uniqueness of the fixed-point $z$ follows easily. Hence, we have the following (see also [10, Corollary 2.1]).

Corollary 2.3. Let $T$ be a self-map of a metric space $X$ and $X$ be T-orbitally complete. Assume that there exists an $x_{0} \in X$ such that for any two elements $x, y \in \overline{O\left(T, x_{0}\right)}$,

$$
\frac{1}{1+k} d(x, T x) \leq d(x, y)
$$

implies the condition (PM.4). Then T has a unique fixed point.

Remark 2.4. Corollary 2.3 generalizes certain theorems from [7, 9-11] and others.

Remark 2.5. It is clear from the proof of Theorem 2.1 that the best value of $\eta$ in class (PM.1)(PM.4) is, respectively, 1/2,1/6,1/4, and $1 / 2$.

The following result is close in spirit to several generalizations of the Banach contraction theorem by Edelstein [22], Sehgal [23], Chatterjea [24], Rhoades [1, conditions (20) and (22)], and Suzuki [15, Theorem 3].

Theorem 2.6. Let $T$ be a self-map of a metric space X. Assume that

(i) there exists a point $x_{0} \in X$ such that the orbit $O\left(T, x_{0}\right)$ has a cluster point $z \in X$,

(ii) $T$ and $T^{2}$ are continuous at $z$,

(iii) for any two distinct elements $x, y \in \overline{O\left(T, x_{0}\right)}$,

$$
\frac{1}{2} d(x, T x)<d(x, y)
$$

implies one of the following conditions:

$(\text { PM.1 })^{*} d(x, T x)+d(y, T y)<2 d(x, y)$,

$(\text { PM.2) })^{*} d(x, T x)+d(y, T y)<(2 / 3)[d(x, T y)+d(y, T x)+d(x, y)]$, 
Fixed Point Theory and Applications

(PM.3)* $d(x, T x)+d(y, T y)+d(T x, T y)<(3 / 2)[d(x, T y)+d(y, T x)]$,

$(\mathrm{PM} .4)^{*} d(T x, T y)<\max \{d(x, y), d(x, T x), d(y, T y),(1 / 2)[d(x, T y), d(y, T x)]\}$.

Then $z$ is a fixed point of $T$.

Proof. An appropriate blend of the proof of Theorems 2.1 and 2 of Pal and Maiti [3] works.

If only the condition (PM.4)* is satisfied in Theorem 2.6, then the uniqueness of the fixed-point $z$ follows easily.

Example 2.7. Let $X=\{0,1 / 4,3 / 4,1\}$ and $T 0=T(1 / 4)=0, T(3 / 4)=T 1=3 / 4$. Then, the map $T$ satisfies all the requirements of Theorem 2.1 with $a=3 / 2, b=7 / 12$, and $k=4 / 5$. Further, $T$ is not a Ćirić-Suzuki contraction, that is, $T$ does not satify the requirements of $[10$, Corollary 2.1] . Evidently, $T$ is not a quasicontraction.

Example 2.8. Let $X=[0,1]$ and

$$
T x= \begin{cases}0, & \text { if } 0 \leq x<\frac{1}{2} \\ \frac{1}{2}, & \text { if } \frac{1}{2} \leq x \leq 1 .\end{cases}
$$

Then, one of the conditions (PM.1)-(PM.4) is satisfied (e.g., $x=49 / 100, y=1 / 2$ ). As $T$ has two fixed points, it cannot satisfy any of the conditions which guarantee the existence of a unique fixed point.

Example 2.9. Let $X=\{3,5,6,7\}$ and

$$
T x= \begin{cases}3, & \text { if } x \neq 6 \\ 6, & \text { if } x=6\end{cases}
$$

Then, the map $T$ satisfies all the requirements of Theorem 2.6. If in Theorem 2.6, the initial choice is $x_{0}=6$ (resp., $x_{0} \neq 6$ ), then $\left\{T^{n} x_{0}\right\}$ converges to 6 (resp., 3 ).

For any subsets $A, B$ of $X, d(A, B)$ denotes the gap between $A$ and $B$, while

$$
\begin{gathered}
\rho(A, B)=\sup \{d(A, B): a \in A, b \in B\}, \\
B N(X)=\{A: \phi \neq A \subseteq X \text { and diameter of } A \text { is finite }\} .
\end{gathered}
$$

As usual, we write $d(x, B)$ (resp., $\rho(x, B)$ ) for $d(A, B)$ (resp., $\rho(A, B)$ ) when $A=\{x\}$.

We use Theorem 2.1 to obtain the following result for a multivalued map.

Theorem 2.10. Let $P: X \rightarrow B N(X)$ and let $X$ be P-orbitally complete. Assume that there exist $a, b, c, k$, and $\eta$ as defined in Section 2 such that for any $x, y \in X$

$$
\eta \rho(x, P x) \leq d(x, y)
$$


implies that at least one of the following conditions is true:

$(\text { PM.1 })^{* *} \rho(x, P x)+\rho(y, P y) \leq a d(x, y)$,

$(\mathrm{PM} .2)^{* *} \rho(x, P x)+\rho(y, P y) \leq b[d(x, P y)+d(y, P x)+d(x, y)]$,

$($ PM.3)** $\rho(x, P x)+\rho(y, P y)+\rho(P x, P y) \leq c[d(x, P y)+d(y, P x)]$,

$(\text { PM.4) })^{* *} \rho(P x, P y) \leq k \max \{d(x, y), d(x, P x), d(y, P y),(1 / 2)[d(x, P y), d(y, P x)]\}$.

Then $P$ has a fixed point.

Proof. It may be completed following Reich [25], Ćirić [2], and Singh and Mishra [11]. However, a basic skech of the same is given below.

Let $\delta=\sqrt{k}$. Define a single-valued map $f: X \rightarrow X$ as follows. For each $x \in X$, let $f x$ be a point of $P x$ such that

$$
d(x, f x) \geq \delta \rho(x, P x)
$$

Since $f x \in P x, d(x, f x) \leq \rho(x, P x)$. So, (2.37) gives

$$
\eta d(x, f x) \leq d(x, y)
$$

and in view of conditions (PM.1) ${ }^{* *}-(\text { PM.4 })^{* *}$, this implies that one of the following is true:

$$
\begin{gathered}
d(x, f x)+d(y, f y) \leq a d(x, y), \\
d(x, f x)+d(y, f y) \leq b[d(x, f y)+d(y, f x)+d(x, y)], \\
d(x, f x)+d(y, f y)+d(f x, f y) \leq c[d(x, f y)+d(y, f x)], \\
d(f x, f y) \leq \frac{k}{\delta} \max \left\{\delta d(x, y), \delta \rho(x, P x), \delta \rho(y, P y), \frac{\delta}{2}[d(x, f y), d(y, f x)]\right\} \\
\leq \sqrt{k} \max \left\{d(x, y), d(x, P x), d(y, P y), \frac{1}{2}[d(x, f y)+d(y, f x)]\right\} .
\end{gathered}
$$

This means Theorem 2.1 applies as " $x, y \in \overline{O\left(T, x_{0}\right)}$ " in the statement of Theorem 2.1 may be replaced by " $x, y \in X$ ". Hence, there exists a point $z \in X$ such that $z=f z$, and $z \in P z$.

\section{Applications}

\subsection{Application to Dynamic Programming}

In this section, we assume that $U$ and $V$ are Banach spaces, $W \subseteq U$ and $D \subseteq V$. Let $\mathbb{R}$ denote the field of reals, $\tau: W \times D \rightarrow W, f: W \times D \rightarrow \mathbb{R}$ and $G: W \times D \times \mathbb{R} \rightarrow \mathbb{R}$. The subspaces $W$ and $D$ are considered as the state and decision spaces, respectively. Then, the problem of dynamic programming reduces to the problem of solving the functional equation

$$
p:=\sup _{y \in D}\{f(x, y)+G(x, y, p(\tau(x, y)))\}, \quad x \in W .
$$


In multistage processes, some functional equations arise in a natural way (cf. Bellman [26] and Bellman and Lee [27]). The intent of this section is to study the existence of the solution of the functional equation (3.1) arising in dynamic programming.

Let $B(W)$ denote the set of all bounded real-valued functions on $W$. For an arbitrary $h \in W$, define $\|h\|=\sup _{x \in W}|h(x)|$. Then, $(B(W),\|\cdot\|)$ is a Banach space. Assume that $\theta(k)=$ $1 /(1+k), 0<k<1$ and the following conditions hold:

(DP.1) $G, f$ are bounded.

(DP.2) Assume that for every $(x, y) \in W \times D, h, q \in B(W)$ and $t \in W$,

$$
\eta(k)|h(t)-K h(t)| \leq|h(t)-q(t)|
$$

implies

$$
\begin{aligned}
& |G(x, y, h(t))-G(x, y, q(t))| \\
& \quad \leq k \max \left\{|h(t)-q(t)|,|h(t)-K h(t)|,|q(t)-K q(t)|, \frac{1}{2}[|h(t)-K q(t)|]+|q(t)-K h(t)|\right\},
\end{aligned}
$$

where $K$ is defined as follows:

$$
K h(x)=\sup _{y \in D}\{f(x, y)+G(x, y, h(\tau(x, y)))\}, \quad x \in W, h \in B(W)
$$

Theorem 3.1. Assume that the conditions (DP.1) and (DP.2) are satisfied. Then, the functional equation (3.1) has a unique bounded solution.

Proof. We note that $(B(W), d)$ is a complete metric space, where $d$ is the metric induced by the supremum norm on $B(W)$. By (DP.1), $K$ is a self-map of $B(W)$.

Pick $x \in W$ and $h_{1}, h_{2} \in B(W)$. Let $\mu$ be an arbitrary positive number. We can choose $y_{1}, y_{2} \in D$ such that

$$
K h_{j}<f\left(x, y_{j}\right)+G\left(x, y_{j}, h_{j}\left(x_{j}\right)\right)+\mu,
$$

where $x_{j}=\tau\left(x, y_{j}\right), j=1,2$.

Further, we have

$$
\begin{aligned}
& K h_{1}(x) \geq f\left(x, y_{2}\right)+G\left(x, y_{2}, h_{1}\left(x_{2}\right)\right) \\
& K h_{2}(x) \geq f\left(x, y_{1}\right)+G\left(x, y_{1}, h_{2}\left(x_{1}\right)\right) .
\end{aligned}
$$

Therefore, (3.2) becomes

$$
\theta(k)\left|h_{1}(x)-K h_{1}(x)\right| \leq\left|h_{1}(x)-h_{2}(x)\right| .
$$


Set

$$
M(k):=k \max \left\{d\left(h_{1}, h_{2}\right), d\left(h_{1}, K h_{1}\right), d\left(h_{2}, K h_{2}\right), \frac{1}{2}\left[d\left(h_{1}, K h_{2}\right)+d\left(h_{2}, K h_{1}\right)\right]\right\} .
$$

From (3.5), (3.7), and (3.8), we have

$$
\begin{aligned}
K h_{1}(x)-K h_{2}(x) & <G\left(x, y_{1}, h_{1}\left(x_{1}\right)\right)-G\left(x, y_{1}, h_{2}\left(x_{1}\right)\right)+\mu \\
& \leq\left|G\left(x, y_{1}, h_{1}\left(x_{1}\right)\right)-G\left(x, y_{1}, h_{2}\left(x_{1}\right)\right)\right|+\mu \\
& \leq k \max \left\{\left|h_{1}\left(x_{1}\right)-h_{2}\left(x_{1}\right)\right|,\left|h_{1}\left(x_{1}\right)-K h_{1}\left(x_{1}\right)\right|,\left|h_{2}\left(x_{1}\right)-K h_{2}\left(x_{1}\right)\right|,\right. \\
& \left.\quad \frac{1}{2}\left[\left|h_{1}\left(x_{1}\right)-K h_{2}\left(x_{1}\right)\right|+\left|h_{2}\left(x_{1}\right)-K h_{1}\left(x_{1}\right)\right|\right]\right\}+\mu \\
& \leq M(k)+\mu .
\end{aligned}
$$

Similarly, from (3.5), (3.6), and (3.8), we get

$$
K h_{2}(x)-K h_{1}(x) \leq M(k)+\mu \text {. }
$$

From (3.10) and (3.11), we have

$$
\left|K h_{1}(x)-K h_{2}(x)\right| \leq M(k)+\mu \text {. }
$$

Since the inequality (3.12) is true for any $x \in W$, and $\mu>0$ is arbitrary, we find from (3.8) that

$$
\theta(k) d\left(h_{1}, K h_{1}\right) \leq d\left(h_{1}, h_{2}\right)
$$

implies

$$
d\left(K h_{1}, K h_{2}\right) \leq M(k) .
$$

So Corollary 2.3 applies, wherein $K$ corresponds to the map $T$. Therefore, $K$ has a unique fixed-point $h^{*}$, that is, $h^{*}(x)$ is the unique bounded solution of the functional equation (3.1).

\subsection{Application to Variational Inequalities}

As another application of Corollary 2.3, we show the existence of solutions of variational inequalities as in the work of Belbas and Mayergoyz [28]. Variational inequalities arise in optimal stochastic control [29] as well as in other problems in mathematical physics, for examples, deformation of elastic bodies stretched over solid obstacles, elastoplastic torsion, and so forth, [30]. The iterative method for solutions of discrete variational inequalities is 
very suitable for implementation on parallel computers with single-instruction, multiple-data architecture, particularly on massively parallel processors.

The variational inequality problem is to find a function $u$ such that

$$
\begin{gathered}
\max \{L u-f, u-\phi\}=0 \quad \text { on } \Omega, \\
u=0 \quad \text { on } \partial \Omega,
\end{gathered}
$$

where $\Omega$ is a nonempty $q$-starshaped open bounded subset of $\mathbb{R}^{N}$ for some $q \in \Omega$ with smooth boundary such that $0 \in \bar{\Omega}, L$ is an elliptic operator defined on $\Omega$ by

$$
L=-a_{i j}(x) \frac{\partial^{2}}{\partial x_{i} \partial x_{j}}+b_{i}(x) \frac{\partial}{\partial x_{i}}+c(x) I_{N}
$$

where summation with respect to repeated indices is implied, $c(x) \geq 0,\left[a_{i j}(x)\right]$ is a strictly positive definite matrix, uniformly in $x$, for $x \in \bar{\Omega}, f$ and $\phi$ are smooth functions defined in $\Omega$ and $\phi$ satisfies the condition: $\phi(x) \geq 0, x \in \partial \Omega$.

The corresponding problem of stochastic optimal control can be described as follows: $L-c I$ is the generator of a diffusion process in $\mathbb{R}^{N}, c$ is a discount factor, $f$ is the continuous cost, and $\phi$ represents the cost incurred by stopping the process. The boundary condition " $u=0$ on $\partial \Omega$ " expresses the fact that stopping takes place either prior or at the time that the diffusion process exists from $\Omega$.

A problem related to (3.15) is the two-obstacle variational inequality. Given two smooth functions $\phi$ and $\mu$ defined on $\bar{\Omega}$ such that $\phi \leq \mu$ in $\Omega, \phi \leq 0 \leq \mu$ on $\partial \Omega$, the corresponding variational inequality is as follows:

$$
\begin{gathered}
\max \{\min [(L u-f, u-\phi), u-\mu]\}=0 \quad \text { on } \Omega . \\
u=0 \quad \text { on } \partial \Omega .
\end{gathered}
$$

Note that the problem (3.17) arises in stochastic game theory.

Let $A$ be an $N \times N$ matrix corresponding to the finite difference discretizations of the operator $L$. We make the following assumptions about the matrix $A$ :

$$
A_{i i}=1, \quad \sum_{j, j \neq i} A_{i j}>-1, \quad A_{i j}<0 \quad \text { for } i \neq j .
$$

These assumptions are related to the definition of "M-matrices", arising from the finite difference discretization of continuous elliptic operators having the property (3.18) under the appropriate conditions and $Q$ denotes the set of all discretized vectors in $\Omega$ (see [31,32]). Note that the matrix $A$ is an $M$-matrix if and only if every off-diagonal entry of $A$ is nonpositive.

Let $B=I_{N}-A$. Then, the corresponding properties for the $B$-matrices are

$$
B_{i i}=0, \quad \sum_{j, j \neq i} B_{i j}<1, \quad B_{i j}>0 \quad \text { for } i \neq j .
$$


Let $b=\max _{i} \sum_{j} B_{i j}$ and $A^{*}$ an $N \times N$ matrix such that $A_{i i}^{*}=1-b$ and $A_{i j}^{*}=-b$ for $i \neq j$. Then, we have $B^{*}=I_{N}-A^{*}$.

Now, we show the existence of iterative solutions of variational inequalities.

Consider the following discrete variational inequalities mentioned above:

$$
\max \left[\min \left\{A\left(x-A^{*} d(x, T x)\right)-f, x-A^{*} d(x, T x)-\phi\right\}, x-A^{*} d(x, T x)-\mu\right]=0,
$$

where $T$ is an operator from $\mathbb{R}^{N}$ into itself implicitly defined by

$$
T x=\min \left[\max \left\{B x+A\left(1-B^{*}\right) d(x, T x)+f,\left(1-B^{*}\right) d(x, T x)+\phi\right\},\left(1-B^{*}\right) d(x, T x)+\mu\right]
$$

for all $x \in \bar{Q}$ such that for all $x, y \in \bar{Q}$, the condition

$$
\theta(k) d(x, T x) \leq d(x, y), \quad \theta(k)=\frac{1}{1+k}, \quad \text { where } k=\max \{b, 1-b\}
$$

holds. Suppose that the condition (3.22) implies that $T$ is defined in $\bar{Q}$ as in (3.21), then (3.20) is equivalent to the fixed-point problem

$$
x=T x,
$$

that is, $\bar{Q} \cap F(T) \neq \emptyset$.

Notice that in two-person game, we have to determine the best strategies for each player on the basis of maximin and minimax criterion of optimality. This criterion will be well stated as follows: a player lists his/her worst possible outcomes, and then he/she chooses that strategy which corresponds to the best of these worst outcomes. Here, the problem (3.20) exhibits the situation in which two players are trying to control a diffusion process; the first player is trying to maximize a cost functional, and the second player is trying to minimize a similar functional. The first player is called the maximizing player and the second one the minimizing player. Here, $f$ represents the continuous rate of cost for both players, $\phi$ is the stopping cost for the maximizing player, and $\mu$ is the stopping cost for the minimizing player. This problem is fixed by inducting an operator $T$ implicitly defined for all $x \in \bar{Q}$ as in (3.21).

Theorem 3.2. Under the assumptions (3.18) and (3.19), a solution for (3.23) exists.

Proof. Let $(T y)_{i}=\left(1-B_{i j}^{*}\right)\left[d\left(y_{i}, T y_{i}\right)+\mu_{i}\right]$ for any $y \in \bar{Q}$ and any $i, j=1,2, \ldots, N$. Now, for any $x \in \bar{Q}$, since $(T x)_{i} \leq\left(1-B_{i j}^{*}\right)\left[d\left(x_{i}, T x_{i}\right)+\mu_{i}\right]$, we have

$$
(T y)_{i}=\max \left\{B_{i j} y_{j}+\left(1-B_{i j}^{*}\right) d\left(y_{i}, T y_{i}\right)+f_{i},\left(1-B_{i j}^{*}\right) d\left(y_{i}, T y_{i}\right)+\phi_{i}\right\}
$$


that is, if the maximizing player succeeds to maximize a cost functional in his/her strategy which corresponds to the best of $N$ worst outcomes from his/her list, then the game would be one-sided. In this situation, we introduce the one sided operator

$$
T^{+} x=\max \left\{B x+A\left(1-B^{*}\right) d(x, T x)+f_{i,}\left(1-B^{*}\right) d(x, T x)+\phi\right\} .
$$

Therefore, we have

$$
(T y)_{i}=\left(T^{+} y\right)_{i}
$$

Now, if $(T x)_{i}=B_{i j} x_{j}+A_{i j}\left(1-B_{i j}^{*}\right) d\left(x_{i}, T x_{i}\right)+f_{i}$, then since

$$
(T y)_{i} \geq B_{i j} y_{j}+A_{i j}\left(1-B_{i j}^{*}\right) d\left(y_{i}, T y_{i}\right)+f_{i}
$$

by using (3.18), we have

$$
\begin{aligned}
\left(T^{+} x\right)_{i}-\left(T^{+} y\right)_{i} \leq & B_{i j}\left\|x_{i}-y_{i}\right\|+\left(1-B_{i j}^{*}\right) \max \left\{d\left(x_{i}, T x_{i}\right), d\left(y_{i}, T y_{i}\right)\right\} \\
\leq & B_{i j}\left\|x_{i}-y_{i}\right\|+\left(1-B_{i j}^{*}\right) \\
& \times \max \left\{d\left(x_{i}, T x_{i}\right), d\left(y_{i}, T y_{i}\right), \frac{1}{2}\left[d\left(x_{i}, T y_{i}\right)+d\left(y_{i}, T x_{i}\right)\right]\right\}
\end{aligned}
$$

If $(T x)_{i}=\left(1-B_{i j}^{*}\right) \cdot d\left(x_{i}, T x_{i}\right)+\phi_{i}$, then since

$$
(T y)_{i} \geq\left(1-B_{i j}^{*}\right) \cdot d\left(y_{i}, T y_{i}\right)+\phi_{i}
$$

we have

$$
\begin{aligned}
(T x)_{i}-(T y)_{i} & \leq\left(1-B_{i j}^{*}\right) \max \left\{d\left(x_{i}, T x_{i}\right), d\left(y_{i}, T y_{i}\right)\right\} \\
& \leq\left(1-B_{i j}^{*}\right) \max \left\{d\left(x_{i}, T x_{i}\right), d\left(y_{i}, T y_{i}\right), \frac{1}{2}\left[d\left(x_{i}, T y_{i}\right)+d\left(y_{i}, T x_{i}\right)\right]\right\}
\end{aligned}
$$

Hence, from (3.18)-(3.20), we have

$$
(T x)_{i}-(T y)_{i} \leq b\|x-y\|+(1-b) \max \left\{d(x, T x), d(y, T y), \frac{1}{2}[d(x, T y)+d(y, T x)]\right\} .
$$

Since $x$ and $y$ are arbitrarily chosen, we have

$$
(T y)_{i}-(T x)_{i} \leq b\|x-y\|+(1-b) \max \left\{d(x, T x), d(y, T y), \frac{1}{2}[d(x, T y)+d(y, T x)]\right\}
$$


Therefore, from (3.31) and (3.32), it follows that

$$
\|T x-T y\| \leq b\|x-y\|+(1-b) \max \left\{d(x, T x), d(y, T y), \frac{1}{2}[d(x, T y)+d(y, T x)]\right\} .
$$

This yields

$$
\|T x-T y\| \leq k \max \left\{d(x, y), d(x, T x), d(y, T y), \frac{1}{2}[d(x, T y)+d(y, T x)]\right\},
$$

where $k=\max \{b, 1-b\}$. Thus, we see that under the assumptions (3.18) and (3.19), for all $x, y \in \bar{Q}$,

$$
\theta(k) d(x, T x) \leq d(x, y)
$$

implies

$$
\|T x-T y\| \leq k \max \left\{d(x, y), d(x, T x), d(y, T y), \frac{1}{2}[d(x, T y)+d(y, T x)]\right\}
$$

Note that $\mathbb{R}^{N}$ is complete and $\bar{Q}$ a closed subset of $\mathbb{R}^{N}$, it follows that $\bar{Q}$ is complete. As a consequence, $\bar{Q}$ is orbitally complete.

Hence, we conclude that all the conditions of Corollary 2.3 are satisfied in $\bar{Q}$. Therefore, Corollary 2.3 ensures the existence of a solution of (3.23).

\section{Acknowledgment}

This research is supported by the Directorate of Research Development, Walter Sisulu University.

\section{References}

[1] B. E. Rhoades, "A comparison of various definitions of contractive mappings," Transactions of the American Mathematical Society, vol. 226, pp. 257-290, 1977.

[2] L. B. Ćirić, "A generalization of Banach's contraction principle," Proceedings of the American Mathematical Society, vol. 45, pp. 267-273, 1974.

[3] T. K. Pal and M. Maiti, "Extensions of fixed point theorems of Rhoades and Cirić," Proceedings of the American Mathematical Society, vol. 64, no. 2, pp. 283-286, 1977.

[4] B. E. Rhoades, "Extensions of some fixed point theorems of Cirić, Maiti, and Pal," Mathematics Seminar Notes. Kobe University, vol. 6, no. 1, pp. 41-46, 1978.

[5] T. Suzuki, "A generalized Banach contraction principle that characterizes metric completeness," Proceedings of the American Mathematical Society, vol. 136, no. 5, pp. 1861-1869, 2008.

[6] A. Abkar and M. Eslamian, "Fixed point theorems for Suzuki generalized nonexpansive multivalued mappings in Banach spaces," Fixed Point Theory and Applications, vol. 2010, Article ID 457935, 10 pages, 2010.

[7] S. Dhompongsa and H. Yingtaweesittikul, "Fixed points for multivalued mappings and the metric completeness," Fixed Point Theory and Applications, vol. 2009, Article ID 972395, 15 pages, 2009. 
[8] M. Kikkawa and T. Suzuki, "Three fixed point theorems for generalized contractions with constants in complete metric spaces," Nonlinear Analysis: Theory, Methods E Applications, vol. 69, no. 9, pp. 2942 2949, 2008.

[9] G. Moţ and A. Petruşel, "Fixed point theory for a new type of contractive multivalued operators," Nonlinear Analysis: Theory, Methods \& Applications, vol. 70, no. 9, pp. 3371-3377, 2009.

[10] O. Popescu, "Two fixed point theorems for generalized contractions with constants in complete metric space," Central European Journal of Mathematics, vol. 7, no. 3, pp. 529-538, 2009.

[11] S. L. Singh and S. N. Mishra, "Coincidence theorems for certain classes of hybrid contractions," Fixed Point Theory and Applications, vol. 2010, Article ID 898109, 14 pages, 2010.

[12] S. L. Singh and S. N. Mishra, "Remarks on recent fixed point theorems," Fixed Point Theory and Applications, vol. 2010, Article ID 452905, 18 pages, 2010.

[13] T. Suzuki, "Some remarks on recent generalization of the Banach contraction principle," in Proceedings of the 8th International Conference on Fixed Point Theory and Its Applications, pp. 751-761, 2007.

[14] T. Suzuki, "Fixed point theorems and convergence theorems for some generalized nonexpansive mappings," Journal of Mathematical Analysis and Applications, vol. 340, no. 2, pp. 1088-1095, 2008.

[15] T. Suzuki, "A new type of fixed point theorem in metric spaces," Nonlinear Analysis: Theory, Methods $\mathcal{E}$ Applications, vol. 71, no. 11, pp. 5313-5317, 2009.

[16] P. C. Bhakta and S. Mitra, "Some existence theorems for functional equations arising in dynamic programming," Journal of Mathematical Analysis and Applications, vol. 98, no. 2, pp. 348-362, 1984.

[17] R. Baskaran and P. V. Subrahmanyam, "A note on the solution of a class of functional equations," Applicable Analysis, vol. 22, no. 3-4, pp. 235-241, 1986.

[18] H. K. Pathak, Y. J. Cho, S. M. Kang, and B. S. Lee, "Fixed point theorems for compatible mappings of type (P) and applications to dynamic programming," Le Matematiche, vol. 50, no. 1, pp. 15-33, 1995.

[19] H. K. Pathak and B. Fisher, "Common fixed point theorems with applications in dynamic programming," Glasnik Matematički, vol. 31(51), no. 2, pp. 321-328, 1996.

[20] H. K. Pathak, S. N. Mishra, and A. K. Kalinde, "Some Gregus type common fixed point theorems with applications," Demonstratio Mathematica, vol. 36, no. 2, pp. 413-426, 2003.

[21] L. B. Cirić, "Generalized contractions and fixed-point theorems," Publications de l'Institut Mathématique, vol. 12(26), pp. 19-26, 1971.

[22] M. Edelstein, "On fixed and periodic points under contractive mappings," Journal of the London Mathematical Society, vol. 37, pp. 74-79, 1962.

[23] V. M. Sehgal, "On fixed and periodic points for a class of mappings," Journal of the London Mathematical Society, vol. 5, pp. 571-576, 1972.

[24] S. K. Chatterjea, "Fixed-point theorems," Comptes Rendus de l'Académie Bulgare des Sciences, vol. 25, pp. 727-730, 1972.

[25] S. Reich, "Fixed points of contractive functions," Bollettino della Unione Matematica Italiana, vol. 5, pp. 26-42, 1972.

[26] R. Bellman, Methods of Nonliner Analysis. Vol. II, Mathematics in Science and Engineering, Vol. 61-II, Academic Press, New York, NY, USA, 1973.

[27] R. Bellman and E. S. Lee, "Functional equations in dynamic programming," Aequationes Mathematicae, vol. 17, no. 1, pp. 1-18, 1978.

[28] S. A. Belbas and I. D. Mayergoyz, "Applications of fixed-point methods to discrete variational and quasivariational inequalities," Numerische Mathematik, vol. 51, no. 6, pp. 631-654, 1987.

[29] A. Bensoussan and J.-L. Lions, Applications des inéquations variationnelles en contrôle stochastique, Méthodes Mathématiques de l'Informatique, no. 6, Dunod, Paris, France, 1978.

[30] G. Duvaut and J.-L. Lions, Inequalities in Mechanics and Physics, Grundlehren der Mathematischen Wissenschaften, 21, Springer, Berlin, Germany, 1976.

[31] A. Berman and R. J. Plemmons, Nonnegative Matrices in the Mathematical Sciences, Computer Science and Applied Mathematics, Academic Press, New York, NY, USA, 1979.

[32] R. S. Varga, Matrix Iterative Analysis, Prentice-Hall, Englewood Cliffs, NJ, USA, 1982. 